\title{
A Randomized Clinical Trial of Attempted Suicide Short Intervention Program versus Crisis Counseling in Preventing Repeat Suicide Attempts: A Two-Year Follow-Up Study
}

\author{
Petri Arvilommi ${ }^{a} \quad$ Jukka Valkonen ${ }^{b}$ Lars H. Lindholm ${ }^{c, d}$ Selma Gaily-Luoma ${ }^{a}$ \\ Kirsi Suominen ${ }^{\mathrm{e}}$ Outi M. Ruishalme ${ }^{\mathrm{b}}$ Marena Kukkonen ${ }^{\mathrm{b}}$ Harri Sihvolab \\ Erkki Isometsäa \\ aDepartment of Psychiatry, University of Helsinki and Helsinki University Hospital, Helsinki, Finland; bMIELI Mental \\ Health Finland, Helsinki, Finland; 'Department of Psychiatry, South Ostrobothnia Hospital District, Seinäjoki, \\ Finland; ' Faculty of Medicine and Health Technology, Tampere University, Tampere, Finland; eHelsinki City \\ Department of Social Services and Healthcare, Psychiatric and Substance Abuse Services, Helsinki, Finland
}

\section{Keywords}

Suicide attempt · Randomized controlled trial · ASSIP .

Effectiveness

\begin{abstract}
Introduction: The Attempted Suicide Short Intervention Program (ASSIP) is a brief psychotherapeutic intervention, and a pivotal study found it to be remarkably effective in reducing repeat suicide attempts. Objective: To compare the effectiveness of ASSIP to crisis counseling (CC) in a randomized clinical trial (ISRCTN13464512). Methods: Adult patients receiving treatment for a suicide attempt in a Helsinki City general hospital emergency room in 2016-2017 were eligible to participate. We excluded psychotic or likely non-adherent substance-abusing or substance-dependent patients. Eligible patients ( $n=239$ ) were randomly allocated to one of two interventions. (a) ASSIP comprised three visits, including a videotaped first visit, a case formulation, and an individualized safety plan, plus letters from the therapist every 3 months for 1 year, and then, every 6 months for the next year. (b) CC typically involved 2-5 (median 3) face-to-face
\end{abstract}

karger@karger.com www.karger.com/pps

Karger $\frac{1}{6}$
(C) 2022 The Author(s)

Published by S. Karger AG, Basel

This is an Open Access article licensed under the Creative Commons Attribution-NonCommercial-4.0 International License (CC BY-NC) (http://www.karger.com/Services/OpenAccessLicense), applicable to the online version of the article only. Usage and distribution for commercial purposes requires written permission. individual sessions. In addition, all participants received their usual treatment. One and 2 years after baseline, information related to participants' suicidal thoughts and attempts, and psychiatric treatment received was collected via telephone and from medical and psychiatric records. Results: Among randomized patients, two-thirds initiated either ASSIP ( $n=$ $89)$ or CC $(n=72)$, with 73 (82\%) completing ASSIP and 58 (81\%) CC. The proportion of patients who attempted suicide during the 2-year follow-up did not differ significantly between ASSIP and CC (29.2\% [26/89] vs. 35.2\% [25/71], OR 0.755 [95\% Cl 0.379-1.504]). Conclusions: We found no difference in the effectiveness of the two brief interventions to prevent repeat suicide attempts. 02022 The Author(s).

Published by S. Karger AG, Basel

\section{Introduction}

A history of attempted suicide is the single most important indicator of risk for suicide, with an accumulated suicide mortality of at least 7-13\% [1-6]. It is, therefore, important to develop interventions aimed at reducing the 
risk of future suicide attempts and deaths. Numerous psychosocial interventions have been developed and investigated, with cognitive behavioral psychotherapies best documented for effectiveness [7-10]. However, although most of these interventions are relatively brief in duration, they may be too resource demanding, limiting their feasibility as a part of routine services. Thus, a great demand exists for brief yet effective interventions aimed at reducing the risk of suicidal acts.

The Attempted Suicide Short Intervention Program (ASSIP) [11] represents a promising brief three-visit intervention. The pivotal randomized controlled study was conducted in Bern, Switzerland, by ASSIP's developers [12], which revealed a remarkable effectiveness in reducing the risk of repeat suicide attempts when compared with treatment-as-usual (TAU) after an index suicide attempt ( 8.3 vs. $26.7 \%$ ) during a 2 -year follow-up period. The intervention also resulted in less use of psychiatric services during the follow-up [12]. However, to our knowledge, these highly promising findings have not been replicated, nor has the effectiveness of ASSIP been compared to another brief intervention. Such a comparison would prove useful with regard to the potential superior and specific effectiveness of ASSIP in reducing suicidal acts.

Therefore, the present study aimed to investigate the effectiveness of ASSIP in reducing repeat suicide attempts over the subsequent 2 years following an index suicide attempt compared to crisis counseling (CC). We hypothesized that the brief ASSIP intervention is superior to CC in reducing repeat suicide attempts, also resulting in lower severity of suicidal ideation and less use of psychiatric services during a 2-year follow-up.

\section{Materials and Methods}

\section{Trial Design and Power Calculation}

We conducted a Zelen randomized, parallel-arm, prospective intervention study to compare the effectiveness of ASSIP and CC in reducing the proportion of new suicide attempts during a 2-year follow-up among patients who had already attempted suicide. Figure 1 illustrates the study design. The study was approved by the local ethics committee and registered as a clinical trial (ISRCTN13464512). All patients signed informed consent forms that met the Declaration of Helsinki guidelines [13]. No financial compensation was paid for participation, although participants received movie ticket gift cards for participating in the 1- and 2-year follow-up telephone interviews.

The pivotal ASSIP study [12] found an absolute risk reduction of $18.4 \%$ between study groups. In the power calculation for the present study, the proportion of patients repeating suicide attempt during follow-up in the CC group was expected to reach approximately $25 \%$, reduced by at least $50 \%$ in the ASSIP group - that is,
$12.5 \%$ or lower. With an $\alpha$ error of 0.05 and $\beta$ error of 0.80 , we estimated that we would need a minimum of 240 patients for randomization in order to detect an absolute difference of $\geq 12.5 \%$ in the repetition rates.

\section{Participants}

The inclusion criteria were (a) a recent suicide attempt, (b) fluency in the Finnish language, and (c) age $\geq 18$ years during the year of inclusion. We excluded patients if they exhibited (a) psychotic features or (b) substance abuse or dependence estimated as sufficiently severe to hamper treatment participation. The recruitment period began on 1 September 2016 and ended on 31 December 2017.

Patients were primarily (a) directly recruited from the emergency rooms of two Helsinki City general hospitals (Haartman and Malmi Hospitals). In addition, (b) some patients were recruited from the psychiatric day-hospital or mood disorder inpatient wards of the Helsinki City Psychiatric Hospital (Aurora Hospital). Additionally, and (c) the acute psychiatric outpatient clinics of Helsinki City referred some individual patients to the study. However, the collaborating units were instructed that only patients who had recently visited an emergency room following a suicide attempt would be eligible to participate in the study.

We defined suicide attempt as in the pivotal study by GysinMaillart et al. [12], as a "self-inflicted, potentially injurious behavior with a nonfatal outcome for which there is evidence (either explicit or implicit) of intent to die."

\section{Randomization}

We randomized patients using Zelen's design, that is, a postrandomization consent design [14]. This randomization design was necessary because the study was conducted within the routine patient pathways of MIELI Mental Health Finland clinical services.

Initially, a patient's contact information was received from the referring unit. The staff member who received the information contacted patients by phone and preliminarily checked their suitability for the study in terms of the inclusion and exclusion criteria, including confirming that a suicide attempt had occurred. If the patient was considered suitable for participation, a sequential number was allotted to the patient. The staff member forwarded the number to the research assistant, who had a list with the sequential numbers randomized through a randomizer program (Research randomizer, www.randomizer.org) either to the ASSIP or CC intervention group. The numbers were drawn in advance in blocks of 30 and assigned to either ASSIP or CC. According to whether the sequential number indicated the ASSIP or CC group assignment for the patient, a respective employee contacted the patient to agree upon an initial appointment.

Patients were asked for their informed consent to participate in the study at the beginning of their initial meeting with a therapist. Thus, informed consent was received only after randomization, and the patient was aware of their group allocation and their therapist when deciding upon participation. Patients who declined to participate knew that they would, nevertheless, receive the same brief intervention to which they had been randomized with the same therapist.

\section{Interventions}

Both brief interventions studied were provided by professionals employed by the Crisis Centre facilities of MIELI Mental Health Finland situated in Helsinki, Finland. 


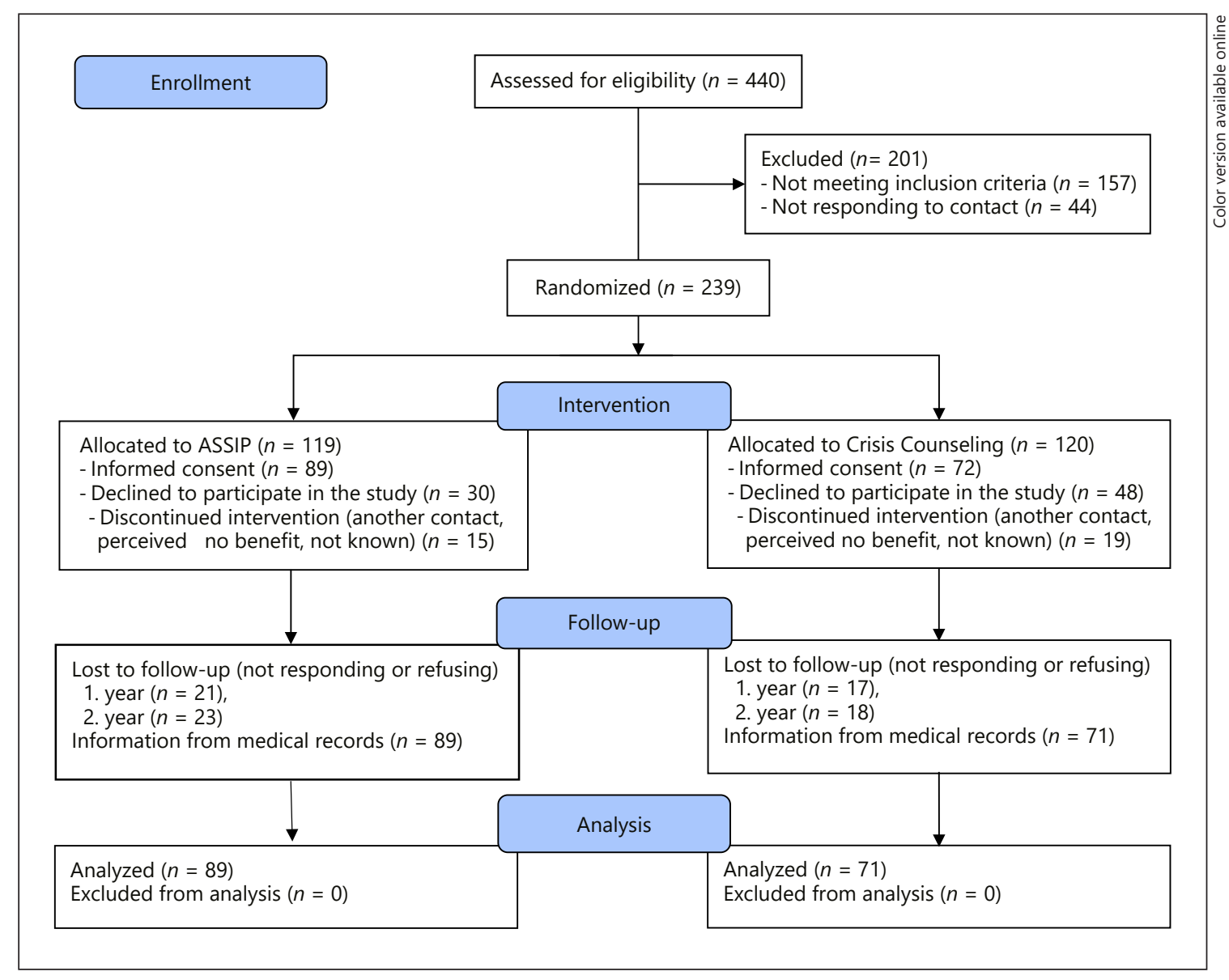

Fig. 1. Flow diagram of the study.

The Attempted Suicide Short Intervention Program

Those in the ASSIP intervention group received the manualized ASSIP brief psychotherapeutic intervention [11]. This consisted of three 60- to 90-min visits, with an optional fourth visit [12]. The ASSIP therapists (M.K., H.S., and J.L.) were trained by the developers of ASSIP - Konrad Michel, MD, and Anja GysinMaillart, PhD - who also provided supervision during the study. Before the study began in 2016, all therapists had clinically provided ASSIP services from 2013-2014 onwards.

The first visit involved a videotaped narrative interview with the patient describing in their own words the sequence of events leading to the suicide attempt. During the second visit, the therapist and patient together watched the video-recorded first session. The video was interrupted for reflections and to discuss additional information. Automatic thoughts, emotions, physiological changes, and contingent behaviors were identified. Patients received a psychoeducational handout ("Suicide is not a rational act") as a homework task. Following the session, the therapist prepared a written draft of the case formulation.

During the third visit, the patient's comments on the handout were discussed. In addition, the written case formulation, warning signs, and safety strategies for possible future crises were completed collaboratively with the patient. The written case formulation and the personal safety strategies were printed for the patient, with additional copies made for other healthcare professionals involved in the treatment. Long-term goals, warning signs, and safety strategies were copied to a credit card-sized folded leaflet and given to the patient. In addition, participants received a crisis card with the telephone numbers for private and professional assistance, which could be contacted in the event of a suicidal crisis. Patients were instructed to carry both items with them at all times and to use them in the event of a crisis. An optional fourth visit was offered to 2 patients $(1.7 \%)$.

Participants also received standardized letters from their therapist every 3 months for 1 year and then, every 6 months for the second year [11]. The letters were signed by the therapist and could be complemented with a personalized sentence. Their aim was to continue the therapeutic contact and remind of safety strategies.

\section{Crisis Counseling}

Participants in the CC group received a course of CC, consisting of meetings with one of six trained counsellors for face-to-face individual treatment lasting $60 \mathrm{~min}$ each. The number of sessions varied from patient to patient as needed, but in $89 \%$ of cases reached $\leq 5$ (median 3 , mean $4.4 ; 8$ individuals had $6-14$ sessions). The crisis counsellors were instructed to provide counseling as usual. 
CC is a conversational intervention, with no specified theory, provided by professionals to support people in crises. We retrospectively surveyed the practices crisis counsellors applied whilst intervening with suicidal clients. The following elements also typical of ASSIP were identified from the crisis counsellors' practices: functional chain analysis, identification of warning signs, and establishing safety strategies. However, these practices were not applied systematically. No counsellor reported applying the elements specific to ASSIP, including the video recordings, transcribing the client's narrative, distributing psychoeducation pamphlets, or following up with letters to patients.

\section{Outcomes}

Primary outcome

The primary outcome was the proportion of patients attempting suicide during the 2-year follow-up period following the first treatment session based on all available information.

\section{Secondary Outcomes}

Secondary outcomes included (1) the total number of repeat suicide attempts (investigated both via telephone interview and from medical and psychiatric records); (2) the severity of suicidal ideation, measured using the Columbia Suicide Severity Rating Scale (C-SSRS) [15], assessed via telephone interviews at 1- and 2-year follow-up time points; and (3) the use of psychiatric and other healthcare services collected from the psychiatric and healthcare records of the City of Helsinki Services.

\section{Data Collection}

Baseline

At baseline, the study participants completed a set of questionnaires. These included a questionnaire to collect sociodemographic and health-related data, including information on previous suicidal behavior and treatments received. We also collected information on prior suicide attempts, psychiatric care, psychotherapy, and medication before baseline. In addition, participants completed scales measuring anxiety (OASIS) [16], alcohol misuse (AUDIT) [17], depressive symptoms (PHQ-9) [18], borderline personality disorder features (MSI-BPD) [19], hopelessness (HS) [20], perceived social support (PSSS-R) [21], suicidal ideation (C-SSRS), and therapeutic alliance (Penn Helping Alliance Questionnaire Method [HAq]) [22].

\section{Therapeutic Alliance}

We measured treatment alliance, a potential moderator of treatment effects [12], using the 11-item HAq after the first, third, and last (if $>$ three) sessions. We found no significant differences in alliance scores between the intervention groups after the first $(t(156)$ $=1.440, p=0.152)$ or last sessions $(t(120)=1.190, p=0.237)$, nor in last post-session scores after adjusting for the first post-session scores $(F=0.368, p=0.545$, partial eta squared $=0.003)$.

\section{Follow-Up}

Follow-up was completed via telephone interviews 1 and 2 years after baseline assessment. During the telephone interviews, possible new suicide attempts as well as attendant and other treatments received during the preceding 12 months were assessed. In addition, all symptom scales were repeated and possible changes in living conditions were examined.

The repeat suicide attempt rate was measured via telephone interviews 1 and 2 years after baseline. The medical and psychiatric records, if any, were also examined for repeat attempts. Since the data could be contradictory and some patients were lost to followup during telephone interviews, the two sources of data were integrated into a total best estimate of suicide attempts during followup.

The psychiatric diagnoses and medications prescribed at the time of the index suicide attempt were assessed from medical and psychiatric records by an experienced psychiatrist (P.A.). Medications prescribed during the 2-year follow-up were also assessed from the medical and psychiatric records.

In addition to the original study protocol, the index suicide attempt, preceding attempts, and repeat attempts during the followup were cross-checked from medical and psychiatric records by a psychologist experienced in suicidology (S.G.-L.). All unclear attempts were assessed within the study group to determine if they satisfied the study criteria.

\section{Blinding}

Patients and therapists in both treatment groups were aware of the arm they were allocated to, although outcome assessors who did the telephone interviews and researchers analyzing the medical and psychiatric records remained blinded to treatment allocation.

\section{Participants}

Altogether, 440 patients were considered potentially eligible and referred to MIELI Mental Health Finland crisis services by referring general hospitals and other facilities. Amongst these patients, 201 either did not meet the inclusion criteria $(n=157)$ or did not respond to our initial contact $(n=44)$.

The remaining 239 patients were identified as eligible for the study and randomized into either of the two interventions. Amongst those eligible and randomized, 161 (67.4\%) provided their informed consent to participate in the study and, thus, comprised the intent-to-treat (ITT) study group (Fig. 1).

In total, 123 participants ( $76.4 \%$ of baseline participants) completed the 1-year follow-up interview and 120 participants $(74.5 \%$ of the baseline sample) completed the 2-year follow-up interview. Complete data across all measurement points were obtained from $63.4 \%$ of the baseline sample (102 participants).

Medical records were available for 160 (99.4\%) patients and reviewed after completion of the telephone interviews. However, when we retrospectively evaluated the index suicide attempts from the medical records after the 2-year follow-up interview, we unexpectedly found 26 patients who did not unequivocally fulfil our inclusion criterion of a recent index suicide attempt (we will describe this sample in a separate article). We carried out separate sensitivity analyses excluding these patients.

We identified no differences in the baseline patient demographics and clinical characteristics for the two intervention groups (Table 1).

\section{Statistical Methods}

In the analyses, we used ITT samples for patients who provided their informed consent to participants in the study. When comparing sociodemographic and clinical factors between the ASSIP and CC groups, we used the $\chi^{2}$ test for categorical data and the MannWhitney $U$ test for continuous variables. To compare the alliance scores between ASSIP and CC groups, we used an independent samples $t$ test and ANCOVA. The primary outcome was assessed using logistic regression analysis. Kaplan-Meier curves and log- 
Table 1. Baseline sociodemographic and clinical characteristics of study participants

\begin{tabular}{|c|c|c|}
\hline Characteristic & $\operatorname{ASSIP}(n=89)$ & $\mathrm{CC}(n=72)$ \\
\hline Age, mean (SD), years & $32.2(13.3)$ & $32.0(12.4)$ \\
\hline Women/men, $n(\%)$ & $62(69.7) / 27(30.3)$ & $52(72.2) / 20(28.7)$ \\
\hline \multicolumn{3}{|l|}{ Diagnosis (ICD-10), $n(\%)$} \\
\hline F1x Substance abuse disorders & $18(20.2)$ & $19(26.4)$ \\
\hline F3x Mood disorders & $75(84.3)$ & $59(81.9)$ \\
\hline F31 Bipolar disorder & $7(7.9)$ & $9(12.5)$ \\
\hline F32-39 Major depressive and other mood disorders & $69(77.5)$ & $50(69.4)$ \\
\hline F4x Anxiety, dissociative, stress-related, somatoform and other & & \\
\hline nonpsychotic mental disorders & $28(31.5)$ & $22(30.6)$ \\
\hline F6x Disorders of adult personality and behavior & $22(24.7)$ & $22(30.6)$ \\
\hline Other & $7(7.9)$ & $3(4.2)$ \\
\hline \multicolumn{3}{|l|}{ Prior suicide attempts, $n(\%)$} \\
\hline 0 & $35(39.3)$ & $34(47.2)$ \\
\hline 1 & $14(15.7)$ & $15(20.8)$ \\
\hline 2 or more (multiple) & $40(44.9)$ & $23(31.9)$ \\
\hline \multicolumn{3}{|l|}{ Psychotropic drugs used, $n$ (\%) } \\
\hline Antidepressants & $42(47.2)$ & $32(44.4)$ \\
\hline Mood stabilizers & $6(6.7)$ & $6(8.3)$ \\
\hline Antipsychotics & $2(2.2)$ & $0(0.0)$ \\
\hline Tranquilizers & $3(3.4)$ & $1(1.4)$ \\
\hline Other hypnotic or sedative medications & $6(6.7)$ & $6(8.3)$ \\
\hline Other & $4(4.5)$ & $1(1.4)$ \\
\hline \multicolumn{3}{|l|}{ Work status, $n(\%)$} \\
\hline Working or student & $52(58.4)$ & $39(54.2)$ \\
\hline Unemployed & $12(13.5)$ & $12(16.7)$ \\
\hline On sick leave or pension & $22(24.7)$ & $16(22.2)$ \\
\hline Other & $3(3.4)$ & $5(6.9)$ \\
\hline Living alone, $n(\%)$ & $43(48.3)$ & $40(55.6)$ \\
\hline Has children, $n(\%)$ & $12(13.5)$ & $9(12.5)$ \\
\hline Lifetime psychiatric hospital treatment, $n(\%)$ & $35(39.3)$ & $27(37.5)$ \\
\hline OASIS sum, mean (SD) & $11.4(4.3)$ & $12.8(3.8)$ \\
\hline PSSS-R sum, mean (SD) & $42.0(11.6)$ & $41.4(11.0)$ \\
\hline AUDIT sum, mean (SD) & $11.7(7.9)$ & $11.5(8.5)$ \\
\hline PHQ-9 sum, mean (SD) & $15.8(6.7)$ & $17.1(6.4)$ \\
\hline MSI-BPD sum, mean (SD) & $6.8(2.2)$ & $7.1(2.1)$ \\
\hline HS sum, mean (SD) & $12.2(5.6)$ & $12.1(5.0)$ \\
\hline C-SSRS sum, mean (SD) & $2.9(2.1)$ & $2.9(1.8)$ \\
\hline
\end{tabular}

rank tests were also used to examine group differences. In addition, we used logistic regression models for multiple sensitivity analyses focusing on specific subgroups or sources of data and, given the potential moderating role of preceding suicide attempts, interaction of the treatment group with this variable. Differences in the number of repeat suicide attempts between the ASSIP and CC groups were analyzed using the Mann-Whitney U test. The difference in the C-SSRS suicide ideation scores between the time points were analyzed using the Friedman's test, and the difference between the ASSIP and CC groups at the time points were analyzed using the $\chi^{2}$ test. We used ANCOVA for analyzing differences in the C-SSRS scores between the intervention groups at 2 years adjusted for baseline scores. Differences in the use of psychiatric and other healthcare services between the ASSIP and CC groups were analyzed using the $\chi^{2}$ test for categorical variables and the MannWhitney $\mathrm{U}$ test for continuous variables.

\section{Results}

\section{Primary Outcome}

Proportion of Patients with Repeat Suicide Attempts

Our primary finding was that there was no significant difference between ASSIP and CC patients who had at least one suicide attempt during the 2-year follow-up (29.2\% [26/89] vs. 35.2\% [25/71]) in the logistic regression models adjusted for age and sex (Table 2; see also the Kaplan-Meier curves, Fig. 2). The finding was similar when we conducted multivariate logistic regression analyses with the scores of PHQ-9, OASIS, MSI-BPD, HS, and preceding suicide attempts 
Table 2. Logistic regression models analyzing the adjusted effectiveness of ASSIP versus CC in preventing repeat suicide attempts during the 2-year follow-up period

\begin{tabular}{llll}
\hline & \multicolumn{3}{l}{ ASSIP vs. CC } \\
\cline { 2 - 4 } & OR & $p$ & $95 \% \mathrm{Cl}$ \\
\hline 1. Primary outcome & & & \\
Repeat suicide attempt during the 2-year follow-up & 0.757 & 0.426 & $0.381-1.504$ \\
2. Sensitivity analyses & & & \\
Patients with an unclear index attempt not included & 0.694 & 0.335 & $0.331-1.457$ \\
Patients with a personality disorder not included & 0.651 & 0.346 & $0.267-1.589$ \\
Patients with a substance use disorder not included & 0.786 & 0.557 & $0.352-1.755$ \\
Patients who discontinued the intervention not included & 0.879 & 0.747 & $0.401-1.927$ \\
Patients with a suicide attempt before the index attempt & 0.622 & 0.302 & $0.252-1.534$ \\
Patients without a suicide attempt before the index attempt & 0.804 & 0.712 & $0.252-2.564$ \\
Repeat suicide attempts only from phone interviews & 1.217 & 0.679 & $0.480-3.087$ \\
\hline
\end{tabular}

All logistic regression models were adjusted for age and sex.

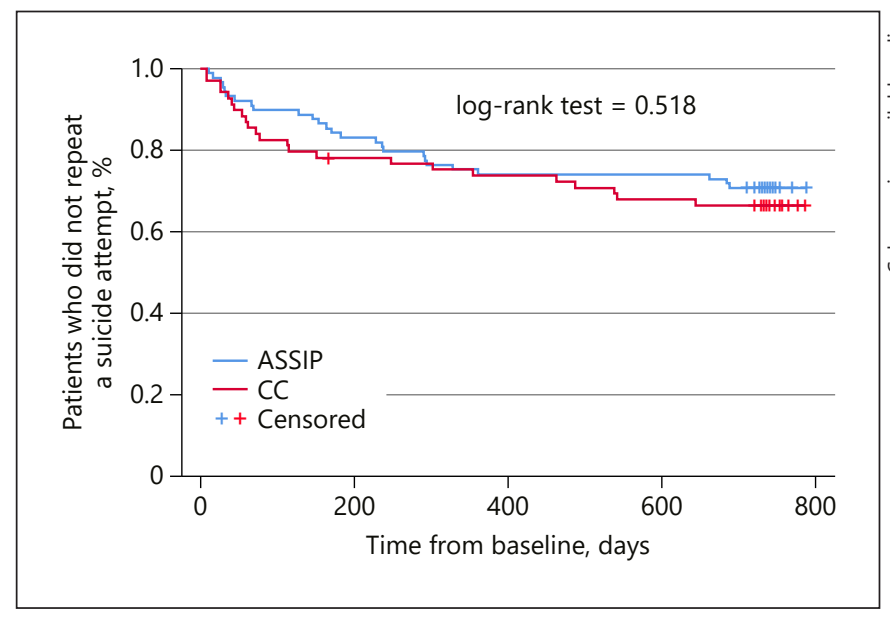

Fig. 2. Kaplan-Meier survival curves of the proportion of patients who did not repeat a suicide attempt during the 2-year follow-up.

as covariates $(\mathrm{OR}=0.715, \mathrm{CI}=0.343-1.492, p=$ 0.371 ).

These results did not change in sensitivity analyses when we excluded patients with an unclear index attempt $(30.7 \%$ [23/75] vs. $39.0 \%$ [23/59]) or patients with a clinical diagnosis of a personality disorder (19.4\% [13/67] vs. $26.5 \%$ [13/49]). In addition, these results remained consistent when we excluded patients with a substance use disorder (26.8\% [19/71] vs. $32.7 \%$ [17/52]), patients who did not complete the interventions $(29.7 \%$ [22/74] vs. $32.1 \%[17 / 53])$ or patients with $(35.2 \%$ [19/54] vs. $45.9 \%$ [17/37]) or without $(20.0 \%$ [7/35] vs. $23.5 \%$ [8/34]) preceding suicide attempts (Table 2 ). Furthermore, a similar
Table 3. Number of repeat suicide attempts during the 2-year follow-up period

\begin{tabular}{lllll}
\hline & \multicolumn{4}{l}{ Number of repeat suicide attempts } \\
\cline { 2 - 5 } & $\begin{array}{l}0 \\
n(\%)\end{array}$ & $\begin{array}{l}1 \\
n(\%)\end{array}$ & $\begin{array}{l}2 \\
n(\%)\end{array}$ & $\begin{array}{l}3 \text { or more } \\
n(\%)\end{array}$ \\
\hline ASSIP,$n=89$ & $63(70.8)$ & $10(11.2)$ & $5(5.6)$ & $11(12.4)$ \\
CC, $n=71$ & $46(64.8)$ & $12(16.9)$ & $6(8.5)$ & $7(9.9)$ \\
\hline
\end{tabular}

analysis of data collected only via telephone interviews revealed no significant difference between the ASSIP and CC groups in terms of the proportion of patients with at least one suicide reattempt $(28.1 \%$ [16/57] vs. $27.3 \%$ [12/44]; Table 2).

Finally, we investigated the possible moderating role of preceding suicide attempts by investigating its interaction with the treatment group (preceding attempts $\mathrm{X}$ treatment group) using logistic regression, finding no evidence for differential responsiveness $(\mathrm{OR}=0.802, \mathrm{CI}=$ $0.185-3.485, p=0.769$ ).

\section{Secondary Outcomes}

Number of Suicide Attempts during the Two-Year Follow-Up

We found no significant difference in the number of suicide attempts during the 2-year follow-up (range: ASSIP 0-26, CC 0-5; mean: ASSIP 1.02, CC 0.73; SD: ASSIP 3.11, CC 1.26; Mann-Whitney $U$ test: $U=3301.00, p=$ 0.556 ; see Table 3 ). 
Severity of Suicidal Ideation

Suicide ideation was measured using the C-SSRS scale for suicidal ideation. We found a steady (mean ASSIP vs. CC: at baseline 2.88 vs. 2.86 ; at 1 year 2.32 vs. 2.15 ; at 2 years 1.39 vs. 1.52 ) and significant reduction in suicide ideation over time from baseline to the 2-year follow-up time point (Friedman test: ASSIP $\chi_{2}^{2}=21.260, p \leq 0.001$; CC $\chi_{2}^{2}=21.168, p<0.001$; combined $\chi_{2}^{2}=41.810, p<$ $0.001)$. However, we found no significant difference in the suicidal ideation scores between ASSIP and CC at baseline $\left(\chi^{2}{ }_{6}=11.347, p=0.078\right)$, at the 1-year $\left(\chi^{2}{ }_{6}=\right.$ $8.223, p=0.222)$ or 2 -year $\left(\chi^{2}{ }_{6}=3.445, p=0.751\right)$ followup time points, nor at 2 years after adjusting for the baseline scores $(F=0.341, p=0.560$, partial eta squared $=$ $0.003)$.

Use of Psychiatric and Other Healthcare Services during the Two-Year Follow-Up

The proportion of patients treated in a psychiatric hospital $(21.7 \%, n=35)$ during the 2-year follow-up period differed $\left(\chi_{1}^{2}=6.535, p=0.011\right)$ between the ASSIP $(26 / 89$, $29.2 \%)$ and CC $(9 / 72,12.5 \%)$ groups, as did the number of days spent in a psychiatric hospital during follow-up (mean/max ASSIP 11.1/235 days vs. CC 2.4/60 days, Mann-Whitney U test $=2,656.00, p=0.010$ ). We detected no difference between ASSIP and CC during follow-up in the number of mental health-based primary care visits (Mann-Whitney $\mathrm{U}$ test $=3,370.00, p=0.510$ ), mental health-based emergency visits (Mann-Whitney $U$ test $=$ $3,245.00, p=0.861)$, nor in the number of psychiatric outpatient visits (Mann-Whitney $\mathrm{U}$ test $=3,026.00, p=$ $0.544)$.

\section{Discussion}

To our knowledge, this represents the first study to compare the effectiveness of the ASSIP intervention to a comparator intervention - in this case, CC - in reducing repeat suicide attempts during a 2 -year follow-up period. Contrary to our hypothesis, we found no difference between the interventions in either primary or most secondary outcomes. The proportion and number of repeat suicide attempts or the level of suicidal ideation during the 2-year follow-up period did not differ between the intervention groups. Thus, taken at face value, our findings do not indicate that ASSIP is specifically more effective than $\mathrm{CC}$ in reducing the risk for repeat suicide attempts.
There are many possible reasons for the differences in findings between our study and the pivotal Swiss study conducted originally by Gysin-Maillart et al. [12]. First, the study designs themselves differ substantially. In order to critically test evidence for the postulated specific benefits, we attempted to control for unspecific factors common to brief psychosocial interventions by comparing ASSIP (+ TAU) to a comparative treatment, CC (+TAU). We chose the comparative treatment both because of the feasibility and given the lack of an explicit specific focus on suicidal thoughts, acts, and safety planning. Thus, using terminology endorsed by Zipfel et al. [23], it served as a specific factor component control. This choice accords with recent discussions on designs of psychotherapy trials overall [23-26]. In contrast, Gysin-Maillart et al. [12] compared ASSIP (+TAU) to TAU alone, without controlling for the nonspecific effects. In their study, patients in both groups received TAU. However, in addition, patients in the TAU group had only one interview with one of the therapists who also delivered ASSIP, whereas patients in the ASSIP group also received the ASSIP intervention. Thus, ASSIP+TAU is likely more effective than TAU alone, but not necessarily specifically more effective than other active brief treatments combined with TAU. On balance, ASSIP was put to a more stringent test than other brief interventions against repetition of suicide attempts, as they have been studied only against TAU, and specificity of effectiveness still remains to be demonstrated $[7,9,27]$.

There are many common factors underlying benefits from all psychotherapies, possibly explaining the majority of the effect in reducing the risk of repeat suicide attempts. Such common factors include therapeutic alliance, empathy, expectations, and cultural adaptation [28]. Given these common factors, it may be as difficult to demonstrate ASSIP's specific superiority compared to standard crisis care as it has been to prove one type of bona fide psychotherapy more effective than other [29]. Notably, we found no difference in the reported therapeutic alliance between the two intervention groups.

We found a small and non-significant numerical absolute risk difference (6\%) in the primary outcome favoring ASSIP. There was no difference in the secondary outcome of suicidal ideation. Contrary to our hypothesis, patients in the ASSIP group received treatment significantly more (not less) often in a psychiatric hospital, although not more psychiatric outpatient treatment than $\mathrm{CC}$ group patients. These significant differences in some but not all forms of help-seeking care, a secondary outcome, could be interpreted as a treatment effect given that 
they are not readily explained by group differences in psychopathology, or as a consequence of suicidal behavior (see Fig. 2). Nevertheless, the exact reasons for these differences remain unclear, and there was no evidence for the ASSIP reducing need of psychiatric services more than CC. It is also important to note that we observed a high rate of repeat suicide attempts. Furthermore, we did not include a third study arm with no treatment or TAU only. Thus, whether the patients received any benefit (or harm) from the two interventions examined remains unknown.

There are also significant contextual differences between the settings in which the studies were conducted. Treatment in the Swiss study was provided in a psychiatric hospital setting, whereas the treatment in our study in Helsinki was provided through a crisis center outside the healthcare service system. The healthcare systems in Switzerland and Finland differ in terms of the availability and funding of services. Thus, there may be significant differences in the standard treatments received. To our knowledge, most of the participants of the Gysin-Maillart et al. [12] trial received some type of psychotherapy after the ASSIP intervention, whereas in Helsinki only a small minority of patients received psychotherapy. It is possible that for some patients the benefits of ASSIP are more effectively maintained or augmented if followed by other psychotherapies. However, in this study, we aimed to examine the comparative effectiveness of two brief interventions, not the role of combining other treatments.

In addition, the patients participating in the studies may have also differed significantly. In our study, patients were younger and more often had clinical diagnoses of affective or personality disorders, whereas they less often presented with anxiety disorders. Thus, the patients in our study seemed to be more severely ill than those in the Swiss study. Furthermore, patients in our study also had slightly more prior suicide attempts than those in the Swiss study. It is likely that patients with more severe disorders or long-term problems do not benefit as much from brief treatment.

When interpreting our findings, we must also consider both its strengths and limitations. This study is the first to compare the effectiveness of ASSIP to another active treatment. Patients were recruited from a group consisting of individuals who attempted suicide treated in two Helsinki City secondary care emergency units, representing the majority of patients attempting suicide and needing emergency treatment. Suicide attempts and the treatment received by patients during the 2-year follow-up were carefully examined, both through interviewing pa- tients and by examining their medical and psychiatric records. The availability of medical and psychiatric records from $99 \%$ of the ITT patients eliminated the risk of attrition biasing our follow-up findings. In addition, those assessing outcomes were blinded to the treatment allocation. The clinical diagnoses were available and carefully checked from the medical and psychiatric records, and we conducted multiple types of sensitivity analyses to confirm the robustness of our findings.

However, we must also outline some significant limitations. First, patients with the medically most severe suicide attempts (i.e., those needing acute surgery or assisted ventilation) were primarily not included since they were treated at Helsinki University Hospital. We also excluded psychotic or severely substance-abusing patients. This influences the generalizability our findings. Second, our study included more patients than the pivotal Swiss study [12], and sampling was based on a power calculation. However, in part because of the post-randomization consent (Zelen) design, our ITT sample size $(n=161)$ reached only two-thirds of the 240 patients originally estimated as necessary to reach a sufficient statistical power, since a significant minority of eligible patients (33\%) declined to participate. Therefore, this lack of a sufficient statistical power (type II error) could, in theory, explain why we found no clinically meaningful differences between the intervention types. However, we find this explanation unlikely, since the observed difference between the interventions was small (absolute risk difference $-6.0 \%$ ), and the findings were quite consistent and robustly persisted in all sensitivity analyses (risk difference ranging from $-10.7 \%$ to $+0.8 \%)$. Nevertheless, we cannot exclude the possibility of minor differences existing in the repetition rates between intervention groups. Third, the proportion of patients refusing participation following randomization was somewhat higher in the CC arm (40.0 vs. $25.2 \%$ ). Thus, in theory, a selection bias may have influenced our findings. However, we detected no significant differences between the study groups in terms of characteristics reported and measured (see Table 1). Fourth, adherence amongst therapists to the ASSIP manual was not formally evaluated, although the therapists were trained and received regular supervision in the intervention and had used it clinically for years before this project began. Fifth, because the therapists in both groups worked in the same facility, some contamination of interventions between therapists may have taken place. This may have somewhat diluted the effect of ASSIP compared to CC. However, none of the therapists in the CC group reportedly videotaped interviews or used any of the other measures 
specific to ASSIP. Sixth, although all of the patients were assessed before inclusion in the study as fulfilling the inclusion criterion of a suicide attempt with an intent to die, unexpectedly some patients appeared not to have met this eligibility criterion following a careful retrospective evaluation of their medical and psychiatric records after the 2 -year follow-up period. However, we conducted a sensitivity analysis excluding these patients, and found no difference in our results. The methodological challenges related to such patients will be reported elsewhere. Seventh, reporting of suicide attempts was not always consistent by patients or in the records, and in cases of discrepant information, we had to rely on researchers' best estimate. However, the researchers were blinded to the treatment allocation, and the findings were robust in sensitivity analyses. Finally, the setting in which the study was conducted, the MIELI Mental Health Finland, is a third-sector organization and not a part of or integrated into the public sector psychiatric care system in Helsinki. Whether researchers' lack of control over the standard treatment provided to their patients influenced patient outcomes remains unknown. This may influence the generalizability of our results to other settings in which ASSIP is provided, particularly in settings where ASSIP is provided as an integral part of comprehensive psychiatric services.

In this randomized study, we did not find that ASSIP was more effective in preventing repeat suicide attempts during a 2-year follow-up when compared with CC.

\section{Acknowledgements}

The authors thank Konrad Michel and Anja Gysin-Maillart for providing instruction on and annual supervision of ASSIP services at MIELI Mental Health Finland from 2013 through 2017.

\section{Statement of Ethics}

This study protocol was reviewed and approved by ethical committee of the Helsinki and Uusimaa Hospital District (HUS), approval number $95 / 13 / 03 / 03 / 2016$, and granted research permits from both the City of Helsinki and the Helsinki and Uusimaa Hospital District. All the participating patients gave written informed consent. The study was conducted in accordance with the Declaration of Helsinki guidelines and registered as a clinical trial (ISRCTN13464512).

\section{Conflict of Interest Statement}

The authors have no conflicts of interest to declare.

\section{Funding Sources}

This study was conducted as part of the crisis services of MIELI Mental Health, Finland, and the study treatments and follow-up were provided without external funding. The researchers received small grants from the Helsinki and Uusimaa Hospital District for purposes of data management, analysis, and writing.

\section{Author Contributions}

Authors of this manuscript comprise the research group responsible for this study. P.A. analyzed patient record data, conducted statistical analyses, and drafted the manuscript. J.V. was responsible for the randomization, patient logistics and data management at the MIELI Mental Health, and supervised follow-up interviews. L.H.L. conducted the survey of crisis counseling therapists' practices. S.G.-L. analyzed patient record data. K.S. led the project within the City of Helsinki general hospitals. O.R. led both the ASSIP and the CC services within the MIELI Mental Health and was the initiator of the ASSIP training. M.K. and H.S. are ASSIP therapists. E.I. is the principal investigator of the study, responsible for study design, and supervised data collection, analysis, and writing. All authors have critically evaluated the manuscript and contributed to its final form.

\section{Data Availability Statement}

Due to limitations imposed by the Finnish data protection legislation, research permits, and content of consent provided by the participating patients, data of this study will not be publicly available.

\section{References}

1 Dahlgren KG. Attempted suicides-35 years afterward. Suicide Life Threat Behav. 1977 Summer;7(2):75-9.

2 Jenkins GR, Hale R, Papanastassiou M, Crawford MJ, Tyrer P. Suicide rate 22 years after parasuicide: cohort study. BMJ. 2002 Nov; 325:1155.
3 Suominen K, Isometsä E, Suokas J, Haukka J, Achte K, Lönnqvist J. Completed suicide after a suicide attempt: a 37-year follow-up study. Am J Psychiatry. 2004 Mar;161(3):562-563.

4 Runeson B, Tidemalm D, Dahlin M, Lichtenstein P, Långström N. Method of attempted suicide as predictor of subsequent successful suicide: national long term cohort study. BMJ. 2010 Jul;341:c3222.
5 Probert-Lindström S, Berge J, Westrin ^, Öjehagen A, Skogman Pavulans K. Longterm risk factors for suicide in suicide attempters examined at a medical emergency in patient unit: results from a 32-year follow-up study. BMJ Open. 2020 Oct;10(10): e038794. 
6 Tidemalm D, Långström N, Lichtenstein P, Runeson B. Risk of suicide after suicide attempt according to coexisting psychiatric disorder: Swedish cohort study with long term follow-up. BMJ. 2008 Nov;337:a2205.

7 Hawton K, Witt KG, Salisbury TLT, Arensman E, Gunnell D, Hazell P, et al. Psychosocial interventions following self-harm in adults: a systematic review and meta-analysis. Lancet Psychiatry. 2016 Aug;3(8):740-50.

8 Zalsman G, Hawton K, Wasserman D, van Heeringen K, Arensman E, Sarchiapone M, et al. Suicide prevention strategies revisited: 10year systematic review. Lancet Psychiatry. 2016 Jul;3(7):646-59.

9 Doupnik SK, Rudd B, Schmutte T, Worsley D, Bowden CF, McCarthy E, et al. Association of suicide prevention interventions with subsequent suicide attempts, linkage to follow-up care, and depression symptoms for acute care settings: a systematic review and meta-analysis. JAMA Psychiatry. 2020 Oct;77(10):102130.

10 Hofstra E, van Nieuwenhuizen C, Bakker M, Özgül D, Elfeddali I, de Jong SJ, et al. Effectiveness of suicide prevention interventions: a systematic review and meta-analysis. Gen Hosp Psychiatry. 2020 Mar-Apr;63:127-40.

11 Michel K, Gysin-Maillart A. ASSIP - Attempted Suicide Short Intervention Program: a Manual for Clinicians. Göttingen: Hogrefe Publishing; 2015.

12 Gysin-Maillart A, Schwab S, Soravia L, Megert $\mathrm{M}$, Michel K. A novel brief therapy for patients who attempt suicide: a 24-months follow-up randomized controlled study of the Attempted Suicide Short Intervention Program (ASSIP). PLoS Med. 2016 Mar;13(3): e1001968.
13 Rickham PP. Human experimentation. Code of ethics of the World Medical Association. Declaration of Helsinki. JBMJ. 1964 Jul; 2(5402): 177 .

14 Zelen M. A new design for randomized clinical trials. N Engl J Med. 1979 May;300(22): 1242-5.

15 Posner K, Brown GK, Stanley B, Brent DA, Yershova KV, Oquendo MA, et al. The Columbia-Suicide Severity Rating Scale: initial validity and internal consistency findings from three multisite studies with adolescents and adults. Am J Psychiatry. 2011 Dec; 168(12):1266-77.

16 Norman SB, Cissell SH, Means-Christensen AJ, Stein MB. Development and validation of an Overall Anxiety Severity And Impairment Scale (OASIS). Depress Anxiety. 2006;23(4): 245-9.

17 Saunders JB, Aasland OG, Babor TF, de la Fuente JR, Grant M. Development of the Alcohol Use Disorders Identification Test (AUDIT): WHO Collaborative Project on Early Detection of Persons with Harmful Alcohol Consumption--II. Addiction. 1993 Jun;88(6): 791-804.

18 Kroenke K, Spitzer RL, Williams JB. The PHQ-9: validity of a brief depression severity measure. J Gen Intern Med. 2001 Sep;16(9): 606-13.

19 Zanarini MC, Vujanovic AA, Parachini EA, Boulanger JL, Frankenburg FR, Hennen J. A screening measure for BPD: the McLean Screening Instrument for Borderline Personality Disorder (MSI-BPD). J Pers Disord. 2003 Dec;17(6):568-73.

20 Beck AT, Weissman A, Lester D, Trexler L. The measurement of pessimism: The Hopelessness Scale. J Consult Clin Psychol. 1974 Dec;42(6):861-5.
21 Blumenthal JA, Burg MM, Barefoot J, Williams RB, Haney T, Zimet G. Social support, type A behavior, and coronary artery disease. Psychosom Med. 1987 Jul-Aug;49(4):331-40.

22 Alexander LB, Luborsky L. The Penn Helping Alliance Scales. In: Greenberg LS, Pinsof WM, editors. The Psychotherapeutic Process: A Research Handbook. New York: Guilford Press; 1986. p. 325-66.

23 Zipfel S, Junne F, Giel KE. Measuring success in psychotherapy trials: the challenge of choosing the adequate control condition. Psychother Psychosom. 2020;89(4):195-9.

24 Gold SM, Enck P, Hasselmann H, Friede T, Hegerl U, Mohr DC, et al. Control conditions for randomised trials of behavioural interventions in psychiatry: a decision framework. Lancet Psychiatry. 2017 Sep;4(9):725-32.

25 Guidi J, Brakemeier EL, Bockting CLH, Cosci F, Cuijpers P, Jarrett RB, et al. Methodological recommendations for trials of psychological interventions. Psychother Psychosom. 2018; 87(5):276-84.

26 Holmes EA, Ghaderi A, Harmer CJ, Ramchandani PG, Cuijpers P, Morrison AP, et al. The Lancet Psychiatry Commission on psychological treatments research in tomorrow's science. Lancet Psychiatry. 2018 Mar;5(3):237-86.

27 Nuij C, van Ballegooijen W, de Beurs D, Juniar D, Erlangsen A, Portzky G, et al. Safety planning-type interventions for suicide prevention: meta-analysis. Br J Psychiatry. 2021 Apr;219:419-26.

28 Wampold BE. How important are the common factors in psychotherapy? An update. World Psychiatry. 2015 Oct;14(3):270-7.

29 Cuijpers P, Reijnders M, Huibers MJH. The role of common factors in psychotherapy outcomes. Annu Rev Clin Psychol. 2019 May;15: 207-231. 